\title{
Le rapport des élèves du secondaire aux jeux électroniques ainsi qu'à I'usage scolaire de logiciels ludo-éducatifs
}

\section{François Larose, Stéphane Palm, Vincent Grenon, Abdelkrim Hasni et Yvan Lessard}

Dans cet article, nous présentons les résultats d'une recherche exploratoire menée auprès d'élèves du secondaire au regard de leurs pratiques d'utilisation de l'informatique en contexte ludique. Après avoir fait état de la documentation scientifique en matière d'exposition des jeunes aux jeux électroniques, nous en décrivons le profil d'utilisation domestique. Cette documentation inclut la représentation que les jeunes ont de l'usage de l'informatique en contexte scolaire, y compris le plan ludo-éducatif. Nous faisons ensuite état de leur appréciation de quatre logiciels recommandés à ce titre par le Ministère de l'éducation, du loisir et du sport, puis nous identifions ce qui, du point de vue des jeunes, devrait définir un logiciel de jeu à la fois intéressant et efficace au plan du soutien à l'apprentissage scolaire. Nous terminons en formulant quelques propositions d'intégration des environnements électroniques de jeu en classe afin de réduire les écarts entre le vécu scolaire et les pratiques sociales de référence des élèves.

On a beaucoup écrit sur les méfaits de l'exposition des jeunes d'âge scolaire aux jeux vidéo depuis le début des années 1990 (Ivory, 2001). Parallèlement, l'intérêt de l'intégration pédagogique des technologies informatiques et des technologies de réseau en enseignement fut particulièrement souligné dans la documentation scientifique, que cette intégration s'adresse aux clientèles en difficulté d'adaptation sociale et scolaire ou encore aux clientèles dites régulières (Aleven, Stahl, Schworm, Fischer \& Wallace, 2003; Mistler-Jackson \& Songer, 2000). Enfin, depuis quelques années on assiste à l'émergence d'un corpus de recherches qui suggèrent que la pratique régulière de jeux électroniques par les jeunes d'âge scolaire, sous certaines conditions, pourrait avoir des incidences positives sur le développement de compétences ciblées par l'enseignement et l'apprentissage scolaire (Durkin \& Barber, 2002).

Cependant, la documentation scientifique suggère que l'usage des technologies de l'information et de la communication (TIC) demeure restreinte dans le contexte quotidien de la salle de classe. Les recherches consultées soulignent aussi que lorsque les enseignants recourent à ces technologies, ils le font selon certains schèmes d'appropriation somme toute fort limités. Le recours à l'informatique 
scolaire, en particulier aux technologies de réseau, se limite généralement à l'utilisation de didacticiels dans une perspective de compensation du temps affecté à l'apprentissage par les élèves «à risque» ainsi qu'à la recherche d'informations sur l'Internet dans le cadre de la préparation de cours chez les enseignants et de la réalisation de travaux scolaires chez les élèves (Larose, Grenon \& Palm, 2004; Office for Standards in Education, 2004; Ungerleider \& Burns, 2003). L'utilisation de l'informatique scolaire en contexte ludique demeure, pour sa part, essentiellement limitée aux activités de renforcement et de soutien à la motivation des élèves, notamment dans le cadre "d'activités récompenses» ou encore d'activités de nature occupationnelle dans les temps libres des élèves (Larose, Lenoir \& Karsenti, 2002; Larose, Lenoir, Karsenti \& Grenon, 2002). La fréquence de ce type d'utilisation pédagogique des TIC décroît d'ailleurs avec la progression scolaire, l'utilisation de logiciels ou d'environnements ludiques ou ludo-éducatifs étant nettement plus fréquente au préscolaire et au premier cycle du primaire puis disparaitt quasi-complètement au secondaire (Larose, Grenon \& Palm, 2004).

Dans le cadre de cet article, nous ferons état de résultats d'un recueil de données préliminaires réalisé dans le cadre d'une recherche subventionnée par le Conseil de recherches en sciences humaines du Canada ${ }^{1}$. D'une façon plus particulière, nous examinerons les pratiques d'utilisation quotidienne des jeux électroniques qui caractérisent des adolescents fréquentant l'école secondaire et l'évaluation qu'ils font de logiciels ludo-éducatifs recommandés par le Ministère de l'éducation du Québec pour utilisation en contexte scolaire. Cette séquence de recueil de données, récurrente sur une base de trois années par ailleurs, s'inscrit dans un travail d'enquête plus large visant premièrement à tracer l'évolution des pratiques de recours à l'informatique ainsi qu'aux jeux électroniques par l'ensemble de la population scolaire québécoise, du début du primaire à la fin de la scolarité obligatoire et, deuxièmement, à identifier les représentations et les pratiques d'intégration de l'informatique scolaire de la part des enseignants de ces deux ordres. Ce programme de recherche a fait l'objet d'un financement récurrent de la part du Conseil de recherches en sciences humaines du Canada (CRSH) depuis 1998 ainsi que, plus ponctuellement, de la part du Fonds québécois de recherche sur la société et la culture (FQRSC) depuis 1999.

\section{Le rapport des jeunes aux jeux électroniques et à leur utilisation scolaire: état de la documen- tation scientifique}

Un corpus documentaire émergent suggère que les efforts d'intégration des TIC en milieu scolaire, en tant qu'outils pédagogique et didactique, tendent à n'avoir que peu d'impact sur la qualité et de la quantité des apprentissages réalisés par les élèves ou encore sur la construction de compétences transversales de leur part (Shyu, 1999; Weinberger, Fischer \& Mandl, 2003). Lorsqu'il y a effets reconnus, 
ces derniers sont relativement mitigés et portent essentiellement sur les dimensions d'acquisition de connaissances de base dans certains domaines disciplinaires ou sur l'adoption d'attitudes favorables à la coopération (Hooper, 2003; Kroesbergen \& Van Luit, 2003; Mathes, Torgesen \& Allor, 2001). Les écrits récents tendent à attribuer ce constat à deux causes majeures. D'une part, l'effet du recours aux TIC sur l'apprentissage semble fortement dépendant du profil d'intervention éducative adopté par l'enseignant et, notamment, sur l'emphase mise sur les dimensions de collaboration dans l'apprentissage en contexte de réalisation de projets (Larose, Grenon, Pearson, Morin \& Lenoir, 2004; Lou, Abrami \& d'Apollonia, 2001). D'autre part, l'écart entre le type de média utilisé et son contexte d'utilisation par les élèves en classe et hors du contexte scolaire semble affecter directement l'efficacité du recours à l'informatique à des fins pédagogique (Mumtaz, 2001). Plus l'environnement d'apprentissage se rapproche de celui que les enfants utilisent à la maison ou dans leur environnement social, plus l'effet sur la qualité et sur la quantité des apprentissages scolaires semble s'affirmer. Ainsi, certaines études récentes tendent à démontrer que l'utilisation de plates-formes variées se rapprochant de celles auxquelles les élèves recourent hors de la classe tels les jeux vidéo (X-Box, Nintendo, Play Station, Game Boy, etc.) ou les cédéroms à caractère ludique sur plate-forme informatique, affecte directement et positivement la qualité des apprentissages scolaires réalisés (Margolis, Nussbaum, Rodriguez \& Rosas, 2004; Rosas et al., 2003; Young \& Upitis, 1999).

Ces travaux rejoignent d'ailleurs les conclusions de tierces recherches portant sur la possibilité de transfert sur le plan de l'apprentissage scolaire, de compétences d'ordre intellectuel et méthodologique construites par les jeunes élèves qui recourent de façon régulière aux environnements électroniques de jeu. Les enfants qui utilisent régulièrement des jeux sur console électronique (jeux vidéo) ou sur plate-forme informatique tendent à développer certaines compétences visuo-spatiales directement transférables dans le cadre de l'apprentissage de la lecture, de l'écriture et de certaines dimensions de la mathématique (géométrie) où le rapport à l'espace est fondamental. Ces élèves ont moins tendances que leurs pairs à valoriser des stratégies d'essai et d'erreur en situation de résolution de problèmes et font davantage appel au raisonnement analogique. Ils construisent plus rapidement que d'autres le schème de règle et, selon le type de jeu utilisé, ils sont plus ouverts à la collaboration que leurs pairs qui fréquentent peu ces environnements (Durkin \& Barber, 2002; Hong \& Liu, 2003; Subrahmanyam, Kraut, Greenfield \& Gross, 2000).

Plusieurs recherches nuancent ces résultats selon la nature, le contenu et le contexte dans lequel les jeux électroniques sont utilisés. Les aspects plus négatifs du recours à ces jeux chez les enfants d'âge préscolaire et scolaire sont généralement associés à deux facteurs distincts. D'une part, la nature cumulative du temps qu'ils accordent à l'utilisation de divers médias dont la télévision, la vidéo, les jeux sur plate-forme électronique et, éventuellement l'ordinateur, peut nuire au temps accordé aux tâches scolaires qu'ils doivent effectuer à la maison ainsi 
qu'à leur disponibilité face à l'apprentissage lorsqu'ils sont en classe. D'autre part, l'exposition systématique à des contenus violents peut affecter leurs conduites lorsqu'ils sont exposés à des situations stressantes. Cependant, ces effets sont fortement modérés par un ensemble de variables associées aux dynamiques familiales et, particulièrement, aux attitudes et aux pratiques éducatives parentales (Frick, Christian \& Wooton, 1999; Gentile \& Walsh, 2002; Linebarger, 2000; Strasburger \& Donnerstein, 1999).

Certaines recherches récentes traitant de l'intégration pédagogique des TIC suggèrent que les enseignants manifestent peu d'intérêt pour les pratiques extrascolaires réelles de leurs élèves en matière de recours aux médias électroniques. Bien qu'ils considèrent que les enfants qui disposent d'ordinateurs réseautés à domicile construisent des compétences techno-instrumentales qui peuvent être transférées sur le plan scolaire, les enseignants ne perçoivent que peu de correspondance possible entre les profils d'utilisation privée de l'informatique chez leurs élèves et celle qui en est faite en classe (Larose, Lenoir \& Karsenti, 2002). Par ailleurs, on constate peu d'incitation à recourir aux médias ainsi qu'aux supports logiciels que les élèves utilisent hors classe dans le discours gouvernemental en matière d'intégration des TIC en enseignement. Une recension des écrits récents suggère plutôt que les enseignants, tant en Amérique du Nord qu'en Europe, tendent à adhérer à un discours voulant que l'exposition massive des enfants aux divers médias électroniques domestiques représente essentiellement un facteur de risque de mésadaptation sociale ou scolaire (Grenon, Larose, Couture \& Desforges, 2003; Schott \& Selwin, 2000). Pourtant, le recours pédagogique aux environnements électroniques dans des contextes se rapprochant de ceux que les élèves utilisent dans la vie courante, semble pouvoir soutenir efficacement l'apprentissage scolaire (Klopfer, Yoon \& Rivas, 2004; Rosas et al., 2003; Schwartz, Lederman \& Crawford, 2004).

\section{Méthodologie et présentation des résultats}

Durant le mois de juillet 2004, un échantillon restreint de soixante-six élèves en âge de fréquentation scolaire secondaire dans la région du Montréal métropolitain était convié à réaliser deux tâches. D'une part, les élèves devaient faire l'essai de quatre logiciels ludo-éducatifs ou considéré tels par le Carrefour des ressources didactiques informatisées $\left(\mathrm{CRDI}^{2}\right)$ du ministère de l'Éducation du Québec. D'autre part, dans le cadre d'entrevues de groupes, les élèves devaient apprécier les logiciels mis à l'essai, en identifier la pertinence d'utilisation en contexte scolaire puis décrire les propriétés que devraient revêtir de «bons» logiciels de jeu à vocation éducative. Les quatre logiciels, compatibles avec une plateforme de type IBM-PC, étaient les suivants (cf. tableau 1). 
Tableau 1: Logiciels ludo-éducatifs mis à l'essai et évalués par les élèves

\begin{tabular}{|c|c|c|}
\hline Identification du logiciel & $\begin{array}{l}\text { Relation à l'apprentissage d'une } \\
\text { matière scolaire spécifique }\end{array}$ & Marché ciblé \\
\hline Une fête médiévale & $\begin{array}{l}\text { Oui; Histoire générale }\left(1^{\mathrm{e}} \text { cycle du }\right. \\
\text { secondaire) }\end{array}$ & Scolaire \\
\hline Casino empire & $\begin{array}{l}\text { Non; Compétences transversales et Do- } \\
\text { maines généraux de formation }\left(3^{\mathrm{e}} \text { cycle }\right. \\
\text { du primaire; } 1^{\mathrm{e}} \text { et } 2^{\mathrm{e}} \text { cycles du secon- } \\
\text { daire) }\end{array}$ & Grand public \\
\hline Mystère en archéologie & $\begin{array}{l}\text { Oui; Sciences humaines ( } 2^{\mathrm{e}} \text { cycle du pri- } \\
\text { maire au } 2^{\mathrm{e}} \text { cycle du secondaire) }\end{array}$ & Scolaire \\
\hline Sim City 4 & $\begin{array}{l}\text { Non; Compétences transversales et Do- } \\
\text { maines généraux de formation }\left(2^{\mathrm{e}} \text { cycle }\right. \\
\text { du primaire au } 2^{\mathrm{e}} \text { cycle du secondaire) }\end{array}$ & Grand public \\
\hline
\end{tabular}

\section{Mé thode}

Après avoir complété une fiche d'information générale permettant de recueillir certaines informations relatives à leur profil sociométrique ainsi qu'à leurs pratiques de recours domestique aux environnements électroniques de jeu, les élèves étaient appelés à utiliser durant une période de 90 minutes, chacun des logiciels ludo-éducatifs mentionnés antérieurement. Quatre cohortes distinctes, de seize à dix-sept jeunes chacune, ont donc pu mettre à l'essai puis évaluer un logiciel spécifique à raison d'un logiciel par semaine durant quatre semaines consécutives. Les cohortes étaient ensuite divisées en quatre groupes de quatre ou cinq élèves, chacun d'entre eux participant à une entrevue collective d'une durée moyenne de trente minutes. Ces entrevues portaient sur les dimensions évaluatives précédemment mentionnées.

\section{Échantil Lon}

L'échantillon en est un de convenance fondé sur le volontariat. Il est constitué de 66 élèves fréquentant un camp de jour ${ }^{3}$ situé sur le campus de l'Université de Montréal, durant le mois de juillet 2004. Le camp de jour est libre d'accès mais la clientèle ciblée a priori en est une d'élèves en âge de fréquentation scolaire de la troisième à la cinquième secondaire soit, en général, des adolescents âgés de quatorze à dix-sept ans révolus au 30 septembre de l'année scolaire en cours. Notre échantillon était composé principalement de jeunes filles (62\%) et secondairement de jeunes garçons (38\%). Environ le tiers de l'effectif était âgé de treize ou quatorze ans (31\%), la majorité des participants avait entre quinze et seize ans (54\%) alors qu'une minorité d'entre eux (15\%) avait dix-sept ans ou plus. Plus du trois-quarts de l'effectif fréquentait des institutions scolaires du secteur public (77\%) alors qu'un peu moins du quart (23\%) étudiait dans le cadre d'écoles secondaires privées. La majeure partie (76\%) des élèves fréquentant des 
institutions scolaires publiques provenait de milieux socio-économiques faibles en foi de l'indice de seuil de faible revenu ${ }^{4}$ accordé par le ministère de l'Éducation aux territoires d'implantation des écoles secondaires de référence (Gouvernement du Québec, 2004).

\section{Traitement des données}

Les données recueillies à l'aide du bref questionnaire distribué aux élèves en début d'activité ont été analysées conformément à la structure de distribution des variables catégorielles, nominales et ordinales, qui en caractérisent les items. Pour les fins de cet article, nous avons essentiellement eu recours à deux types de calcul. D'une part, nous avons procédé à l'analyse bivariée de l'interaction entre nos variables de contexte (âge, statut socio-économique des clientèles scolaire, genre) et l'ensemble de variables descriptives disponibles à l'aide du coefficient de vraisemblance (Likelihood ratio chi square). Celui-ci, contrairement au chi carré, est peu sensible à l'effet de marginalisation des fréquences lorsque les échantillons sont numériquement restreints et que le nombre de catégories des variables croisées s'avère inégal (Andersen, 1990).

Pour l'analyse multivariée de ces mêmes variables, notamment lors de la détermination des profils d'utilisation de diverses catégories de jeux vidéo, nous avons procédé à une série d'analyse des correspondances multiples (Heiser \& Meulman, 1994; Meulman, Van der Kooij \& Heiser, 2004). Les algorithmes de calcul des analyses factorielles de correspondance intègrent des routines de contrôle (pondération) de l'effet de surreprésentation ou, au contraire, de sousreprésentation de l'effectif spécifique aux zones d'interaction des catégories de variables croisées. Il y a donc contrôle et réduction d'une source de biais majeur associé à la distorsion qu'entraîne la projection d'une partition multidimensionnelle sur un plan en deux dimensions. Bien que plus complexes et moins précises, des procédures équivalentes s'appliquent au calcul des analyses de correspondances multiples. Enfin, l'analyse des données textuelles recueillies lors des entrevues de groupe a été réalisée selon une approche lexicométrique considérant le mot en tant que variable nominale pouvant faire l'objet d'un croisement avec n'importe quelle variable de contexte induisant suffisamment de variation dans la structure du discours (Giegler \& Klein, 1994; Lebart, Salem \& Berry, 1997). Le modèle statistique utilisé demeure celui de l'analyse factorielle des correspondances 5 .

\section{Le profil de recours aux jeux électroniques et les plates-formes utilisées: éléments descriptifs}

La majeure partie des sujets dispose d'un micro-ordinateur ainsi que d'un accès Internet à la maison. Un peu plus du deux-tiers d'entre eux dispose aussi d'au 
moins une console de jeu vidéo à domicile (cf. tableau 2). Le statut socio-économique des élèves et le fait de disposer d'un micro-ordinateur relié ou non à l'Internet ou d'une console vidéo ne sont pas associés. Cette variable n'est pas associée, non plus, au fait d'utiliser l'ordinateur, réseauté ou non, en tant qu'environnement de jeu privilégié. Il n'y a pas, enfin, d'association significative entre l'âge des sujets et l'une ou l'autre des cinq variables mentionnés.

Tableau 2: Accès domestique à des environnements de soutien au jeu électronique et type d'environnement privilégié

\begin{tabular}{|l|c|c|}
\hline & N & Pourcentage \\
\hline Élèves disposant d'un micro-ordinateur à domicile & 60 & 92 \\
Élèves disposant d'un lien Internet à domicile & 55 & 83 \\
Élèves utilisant un ordinateur pour jouer & 50 & 76 \\
Élèves disposant d'une console de jeu électronique à domicile & 42 & 68 \\
Élèves utilisant l'Internet pour jouer en réseau & 25 & 40 \\
\hline
\end{tabular}

Par contre, le genre est associé au fait d'utiliser l'Internet pour jouer en réseau, les garçons recourant significativement plus fréquemment à cet environnement pour cette fin que les filles $\left(L^{2}=5,63[1] ; p<0,018\right)$. Enfin, ce sont les élèves qui utilisent l'Internet pour jouer avec des tiers en réseau qui ont les pratiques les plus fréquentes de recours aux jeux électroniques $\left(L^{2}=11,002[3] ; p<0,012\right)$. Ces élèves sont ceux qui pratiquent un jeu électronique au moins une fois par jour. Il s'agit généralement de garçons, les filles ayant pour leur part des fréquences de pratique du jeu électronique significativement inférieures à celles de leurs pairs masculins $\left(L^{2}=30,14[3] ; p<0,001\right)$. En fait, elles forment plus du deux-tiers de ceux qui jouent moins de quatre fois par semaine et moins de vingt pourcent de ceux qui jouent plus de cinq fois par semaine.

D'une façon générale, lorsqu'ils ne jouent pas à l'aide d'un ordinateur, nos sujets utilisent une assez grande variété d'environnements de jeu électronique (cf. tableau 3). 
Tableau 3: Types de plate-forme de jeu privilégié, autres que l'ordinateur

\begin{tabular}{|l|c|c|}
\hline & N & Pourcentage \\
\hline Plus d'un type de console & 17 & 38 \\
Playstation & 11 & 25 \\
X Box & 6 & 13 \\
Nintendo & 3 & 7 \\
Game Boy & 3 & 7 \\
Game Cube & 2 & 4 \\
Autre type de plate-forme & 2 & 4 \\
Dreamcast & 1 & 100 \\
\hline Total & 45 & 2 \\
\hline
\end{tabular}

$\mathrm{Si}$ on regroupe ces consoles selon les critères de classification en vigueur (Wikipedia, 2004a) soit qu'il s'agisse de consoles de 128 bits suffisamment puissantes pour soutenir les jeux à haute résolution graphique, des consoles plus anciennes de 64 bits ou encore de consoles portables, nous pouvons observer une nette prédominance des premières (cf. tableau 4). On ne constate aucune structure d'association entre le type de console utilisée et le genre, le statut socio-économique ou l'âge des élèves.

Tableau 4: Types de plate-forme de jeu privilégié, selon la classification des consoles

\begin{tabular}{|l|c|c|}
\hline & N & Pourcentage \\
\hline Consoles fixes de 128 bits & 20 & 44 \\
Plus d'une console & 17 & 38 \\
Consoles de 64 bits & 5 & 11 \\
Consoles portables & 3 & 7 \\
\hline Total & 45 & 100 \\
\hline
\end{tabular}

Dans le questionnaire qui leur a été distribué, nous demandions aux élèves d'identifier par ordre de fréquence, les quatre jeux sur support informatique 
dont ils avaient le plus fréquemment usage. Nous avons reclassé les jeux mentionnés en respectant la classification par genre en vigueur (Wikipedia, 2004b) de façon à restreindre la zone de classement à onze catégories opératoires (cf. tableau 5). Comme le lecteur pourra le constater, ce sont de loin les jeux d'aventure, les jeux de combat et les jeux de sport qui sont les contextes de prédilection des élèves. Le calcul des mesures d'association permet de constater que seules les variables genre et âge des sujets sont associées au type de jeu que pratiquent les sujets. Nous avons donc procédé à une série d'analyses des correspondances multiples croisant ces deux variables, le type d'équipement détenu et l'ensemble des types de jeux dont la fréquence d'usage dépassait un critère arbitraire de $20 \%$ de l'effectif, de façon à tenir compte des limites de capacité de pondération de l'effet des fréquences marginales qui affectent les statistiques dérivées des modèles d'analyse factorielle des correspondances.

Tableau 5: Classification des jeux électroniques utilisés selon le genre

\begin{tabular}{|l|c|c|}
\hline & N & Pourcentage \\
\hline Jeux d'aventure & 37 & 56 \\
Jeux de combat & 21 & 32 \\
Jeux de sport & 21 & 32 \\
Jeux de course & 11 & 17 \\
Jeux de stratégie & 10 & 14 \\
Jeux issus de situations de vie courante & 9 & 9 \\
Jeux de rôle & 6 & 5 \\
Jeux de puzzle & 3 & 2 \\
Jeux musicaux & 1 & 2 \\
Jeux de simulation & 1 & - \\
Jeux éducatifs & - & \\
\hline
\end{tabular}

Comme le lecteur pourra le constater en consultant la figure 1, ce sont essentiellement les garçons âgés de quinze à seize ans qui jouent aux jeux de combat ainsi qu'aux jeux d'aventure. Leurs pairs féminin âgés de dix-sept ans jouent moins fréquemment et, lorsqu'ils le font, pratiquent surtout les jeux de sport. Sauf cette exception, les filles n'utilisent que peu ou pas les trois types de jeux électroniques décrits, indépendamment de l'âge. 


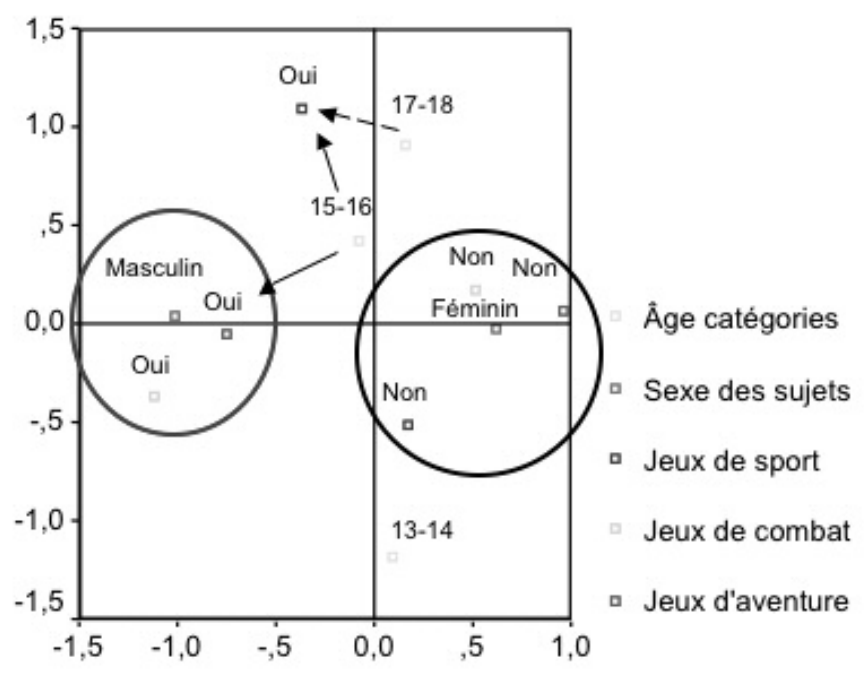

Figure 1: Analyse des correspondances multiples

Variables genre, âge et les trois types de jeux les plus pratiqués

Par ailleurs, les garçons jouent aux jeux de combat et d'aventure en recourant à plus d'un type de console alors que ceux qui pratiquent les jeux de sports tendent à le faire en utilisant les supports les plus puissants soit les consoles de type 128 bits (figure 2).

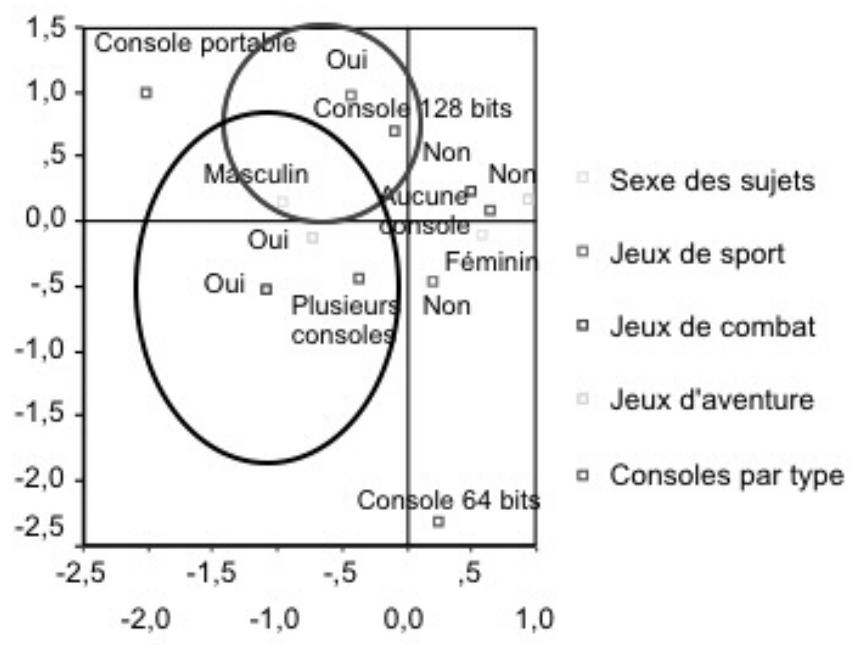

Figure 2: Analyse des correspondances multiples

Variables genre, type de console et les trois types de jeux les plus pratiqués 
Enfin, l'intégration de la variable socio-économique dans le modèle permet de constater que les élèves provenant de milieux défavorisés, âgés de quinze à seize ans, sont ceux qui ont le plus souvent tendance à utiliser les consoles plus puissantes alors que leurs pairs plus âgés, provenant de milieux mieux nantis, sont ceux qui recourent le plus fréquemment à des supports électroniques multiples.

\section{L'analyse du discours des élèves au regard des jeux électroniques testés}

Pour les fins de cet article, nous avons retenu deux des quatre thèmes qui étaient abordé lors des entrevues avec chacun des quatre groupes d'élèves à la fin de chacune des séquences de jeu. Ces thèmes sont les suivants:

- Perception et appréciation du jeu mis à l'épreuve;

- Utilité du recours aux jeux électroniques pour l'apprentissage scolaire.

Appréciation des jeux

Le premier thème laisse entrevoir une assez grande variation dans l'appréciation des divers jeux chez les élèves, tout en permettant de constater une homogénéité importante de l'appréciation entre les quatre équipes pour chacun des jeux mis à l'essai. D’une façon générale les élèves ont apprécié le premier jeu à la fois au plan graphique et sur celui de la simplicité d'utilisation mais s'en sont rapidement lassé. Ce jeu, «Une noce en Nouvelle-France/Une fête médiévale» se fonde essentiellement sur une stratégie de repérage visuel (repérer des objets dans divers tableaux), de rappel puis de recherche et de récupération permettant de disposer ces objets dans un coffre. La recherche des objets est associée à une série de textes à connotation historique qui en servent d'indice. Pour nos sujets, il s'agit de l'archétype du jeu qui pourrait intéresser les élèves d'âge scolaire primaire. La qualité des graphiques est reconnue par l'ensemble des équipes mais les participants critiquent de façon unanime le caractère agaçant de la trame sonore et la qualité non professionnelle des éléments de programmation occasionnant des problèmes liés à la récupération des objets trouvés devant être déplacés dans un coffre et nécessitant plusieurs essais pour y arriver. Par ailleurs, dans la mesure où on tient compte des textes affichables en tant qu'indice, il s'agit pour les élèves d'une tâche scolaire potentiellement intéressante, directement associée aux contenus académiques explorés en histoire au secondaire et non d'un jeu. Autrement dit, en tant que contexte ludique, l'environnement logiciel leur semble correspondre aux attentes et caractéristiques d'enfants beaucoup plus jeunes alors qu'en tant qu'outil didactique, cet environnement leur semble présenter une tâche adéquate pour le soutien à la révision de connaissances dans une matière scolaire particulière de leur niveau.

Le second jeu, Casino Empire, une version contextualisée répondant de la même logique que Sim City 4, a été considéré intéressant par l'ensemble de nos 
jeunes «évaluateurs». L'intérêt décroît cependant relativement rapidement, soit après environ une demie-heure d'utilisation, pour la moitié de l'effectif. Les causes de cette démotivation progressive sont simples. D’une part, les élèves qui ne sont pas déjà familiers avec ce type de jeu n'utilisent pas ou très peu les fonctions d'aide et d'explication offertes par le logiciel. Ils se retrouvent donc rapidement en situation d'échec ou, dans le contexte du jeu, en situation de banqueroute. Ces élèves soulignent l'importance de la fonction de renforcement, le succès à court terme et la progression constante dans la capacité d'augmenter les bénéfices du Casino qu'ils doivent gérer, dans le maintien de leur intérêt au regard du jeu. D'autre part, ces mêmes élèves n'explorent pas les fonctions avancées du logiciel et, en conséquence, ne sont pas en situation de planifier la croissance de l'environnement qu'ils gèrent. Ils ne peuvent situer leur action dans une perspective de développement à long terme donc ne peuvent appliquer de stratégies de résolution de problèmes conséquentes. Leurs pairs qui ont pris le temps de procéder à l'exploration des fonctions de base ainsi que des fonctions avancées du logiciel, qui ont généralement parcouru la fonction de jeu illustratif (démo.), maintiennent un haut niveau de motivation et considèrent que le temps alloué pour la mise en œuvre du logiciel est insuffisant.

Le logiciel leur semble présenter une dimension ludique réelle, adaptée aux besoins et caractéristiques de leur âge. Ce type de jeu informatique leur semble favoriser la mise en œuvre de compétences cognitives associées à la résolution de problèmes. Il pourrait donc s'agir d'une situation de type ludique favorisant l'application contextualisée de compétences d'ordre mathématique. Le contexte même du logiciel, la gestion d'un casino et les stratégies favorisant son développement et son expansion, amène certains élèves à considérer que ce jeu informatique pourrait soutenir le développement de situations de réflexion et de discussion au regard des enjeux et des effets sociaux des jeux de hasard. Il y a là, pour des adolescents, matière à réflexion spontanée portant sur des problématiques sociales importantes dans l'actualité québécoise.

Le troisième jeu, Mystère en archéologie, est perçu par la majorité des élèves comme une tâche scolaire relativement intéressante. Cependant la dimension ludique n'est que peu ou pas perçue de la part de nos sujets. Le logiciel propose à l'élève cinq missions au travers desquelles il devra élucider un mystère, en l'occurrence découvrir l'identité sociale et historique d'un squelette exhumé sur un site de fouilles archéologique de l'Ile de Montréal (Pointe-à-Callière). Pour nos élèves, la relation avec une matière scolaire particulière est difficile à établir, ce qui les amène à considérer que le but du recours à ce jeu est d'explorer les diverses facettes du métier d'archéologue et à inférer, en conséquence, que le logiciel est en fait un outil didactique associé au programme secondaire d'éducation au choix de carrière. Nos jeunes évaluateurs considèrent qu'au plan technique, soit en matière de qualité des graphiques et de relative interactivité, le logiciel est de bonne qualité. Néanmoins, l'importance de l'information écrite pour la réalisation des différentes missions fait en sorte que, pour eux, il ne peut s'agir d'un jeu 
mais plutôt qu'il s'agit d'emblée d'une tâche scolaire de nature relativement indéterminée.

Enfin, le quatrième jeu informatique, Sim City 4, est probablement l'un des jeux de simulation les plus diffusés et sans nul doute l'un des plus populaires à l'échelon international. L'enjeu, comme pour l'ensemble des jeux informatiques de la série des "Sims ", est de gérer le développement et l'administration d'une ville et d'en faire une mégapole. Dans l'ensemble, les élèves ont considéré qu'il s'agissait d'un contexte ludique intéressant. La majeure partie d'entre eux soulignent la qualité des interfaces graphiques et le haut niveau d'interactivité du logiciel. Cependant, dans ce cas comme dans celui de l'expérimentation du Casino Empire, les élèves manifestent une attitude partagée au regard de la possibilité de maintenir un haut niveau de motivation au regard de l'activité. Pour près de la moitié d'entre eux, le nombre de paramètres dont le joueur doit tenir compte afin d'assurer la croissance de sa ville tout en garantissant un niveau de satisfaction suffisant de la part de ses habitants est trop élevé. Or, sans l'exercice de ce contrôle, les joueurs font rapidement face à une situation de stagnation ou de banqueroute qui s'avère démotivante pour plusieurs d'entre eux. Le fait que le jeu s'inscrive dans une perspective de longue durée, les parties pouvant être sauvegardées et reprises au gré du joueur, compense la difficulté précédente pour une minorité de sujets. Encore une fois, plusieurs élèves considèrent que ce jeu représente un lieu d'application potentiel des compétences d'ordre mathématique explorées en contexte scolaire.

\section{Analyse des représentations des élèves au regard de l'utilité du recours aux jeux électroniques pour L'apprentissage scolaire}

Le second thème permet de constater une assez grande variation dans l'appréciation des diverses matières scolaires pour l'apprentissage desquelles le recours aux jeux informatiques semble utile à nos sujets. L'analyse du discours portant sur ce thème permet aussi de constater un clivage assez net chez les élèves au regard de l'utilité même de cette intégration. L'utilité du recours au jeu électronique pour soutenir l'apprentissage scolaire apparaît évident pour la majeure partie de nos sujets, du moins lorsque les clientèles ciblées fréquentent le primaire. À cet effet, les matières scolaires principalement identifiées sont le français (langue maternelle) et la mathématique. Le type de jeux décrit reflète celui auxquels les élèves ont été exposés durant leur parcours scolaire. Il s'agit essentiellement de logiciels ludo-éducatifs ciblant des structures d'apprentissage relativement fondamentales, telle la série "Adibou» ou encore de logiciels proposant des tâches de repérage et d'association du type de «Une noce en Nouvelle-France/Une fête médiévale» dont nous avons précédemment traité.

Dans le cas du français, la quasi totalité des élèves fait mention du fait qu'il s'agit de la seule matière scolaire pour laquelle ils ont une expérience d'exposition systématique à l'utilisation d'un support électronique, notamment dans le cadre de réalisation de travaux de recherche en contexte scolaire. Cependant ce type 
d'utilisation ne présente guère de dimension ludique. Outre ce qui précède, les logiciels ou environnements de jeux téléaccessibles auxquels nos élèves ont eu accès en classe depuis leur passage au secondaire leur semble ne présenter que peu de potentiel au regard de l'apprentissage scolaire. Il ne s'agit d'ailleurs le plus souvent que de logiciels fournis avec l'environnement Windows ou de sites Internet auxquels ils peuvent avoir accès depuis les laboratoires d'informatique de leurs institutions respectives au titre de passe-temps entre deux périodes d'enseignement. Ces jeux, assez sommaires, dépassent rarement le niveau de sophistication du jeu de puzzle, du jeu de cartes ou du jeu de «pinball».

Pourtant la majeure partie des élèves font état du potentiel d'un recours structuré, intégré aux activités didactiques et pédagogiques ayant cours dans le cadre de l'enseignement secondaire, en ce qui concerne certains jeux de grande diffusion. Ils associent ce recours à certaines matières spécifiques telles la mathématique, la géographie et l'histoire. Pour ces élèves le recours à un jeu de stratégie, par exemple "Age of Empires», permet de tenir compte à la fois de compétences et de contenus (connaissances) appropriées à ces trois disciplines, dans une perspective intégrée. Ils considèrent aussi que leurs pratiques informelles de recours aux jeux électroniques appuie le développement de leurs compétences en anglais langue seconde dans la mesure où la majorité des logiciels ludiques auxquels ils recourent sont produit dans cette langue. En conséquence, ils considèrent que l'utilisation scolaire de jeux de stratégies en version originale pourrait fournir un contexte à la fois stimulant et pertinent pour la mise en ouvre de leurs compétences en lecture en langue seconde.

\section{Discussion}

Les résultats que nous avons présenté, indépendamment du caractère restreint de l'échantillon, font largement écho à l'état de la documentation scientifique à plusieurs égards. Ainsi, l'absence d'effet du statut socio-économique sur le fait de posséder une plate forme de jeu électronique ou d'avoir accès à un ordinateur à la maison reflète une tendance émergente du rapport des jeunes d'âge préscolaire et scolaire aux jeux électroniques et à l'informatique domestique (Anand \& Krosnick, 2005; McLelland \& Crawford, 2004). À cet effet, la fracture numérique (digital gap) entre jeunes de milieux socio-économiques distincts semble plutôt se manifester au plan de la connectivité et de l'accessibilité d'Internet à la maison qu'en matière de disponibilité générale des équipements électroniques ou informatiques (Sciadas, 2002; Statistique Canada, 2004a).

Par ailleurs, les différences basées sur le genre et sur l'âge dont nous avons fait état au regard des préférences quant au type de jeu pratiqué ainsi qu'aux fréquences de recours au jeu électronique sont aussi conformes à ce que constatent plusieurs recherches dans ce domaine. Les filles jouent moins fréquemment que les garçons à des jeux électroniques, elles ont moins tendance à jouer en contexte 
d'interaction sociale et aiment moins que leurs pairs masculin les jeux compétitifs impliquant un rapport à des situations réalistes se déployant dans des espaces virtuels complexes (Bickham et al., 2003; Lucas \& Sherry, 2004). Les filles ont aussi moins tendance que leurs pairs masculins à utiliser l'ordinateur à la maison, la différence étant attribuable à une moindre propension au jeu sur ordinateur et à un profil de recours à l'informatique plus axé sur les communications interindividuelles par courrier électronique ou clavardage (chat) chez les filles (Colley \& Comber, 2003).

L'appréciation différenciée de la dimension ludique de certains logiciels en fonction des caractéristiques des élèves d'âge scolaire primaire et d'une lecture à caractère purement «académique» de ce recours au secondaire représente probablement un des éléments particuliers de notre recherche. La question qui semble se poser ici est la suivante: est-ce qu'on peut apprendre en jouant au secondaire? Les élèves de notre échantillon semblent y répondre de façon positive mais lorsqu'ils le font c'est au travers des propriétés de logiciels ludiques de grande diffusion, donc de nature commerciale, et du potentiel qu'ils semblent présenter au regard d'apprentissages associés à des matières scolaires spécifiques. Il y a là deux dimensions particulières qui méritent réflexion.

Premièrement, le fait qu'on associe des propriétés au plan des contenus d'apprentissage ciblés dans certaines matières scolaires à des jeux de stratégie de grande diffusion confirme le résultat de quelques études expérimentales menées en Grande Bretagne. Ces études, notamment celles qui ont été menées dans six écoles secondaires desservant des clientèles régulières ou en difficulté d'adaptation sociale et scolaire, suggèrent des effets positifs du recours aux jeux de stratégie ainsi qu'à certains jeux de sport sur la construction de compétences disciplinaires particulières. On constate aussi des effets chez les élèves de la pratique de ces jeux sur la construction de compétences transversales d'ordre intellectuel directement associées à ce que cible le curriculum britannique (British Educational Communication and Technology Agency, 2001; McFarlane, Sparrowhawk \& Heald, 2002).

Deuxièmement, nos résultats peuvent être interprétés à l'aulne des plus récentes recherches en ce qui concernent les facteurs qui affectent la rapidité et la durabilité des apprentissages réalisés en contexte d'exposition au jeu électronique ainsi que les conditions de succès de ces jeux auprès des adolescents. Pour qu'un jeu électronique soutienne l'attention et la motivation de l'utilisateur, il doit présenter un haut niveau de qualité au plan de la résolution graphique et sonore et offrir une forme d'interactivité s'approchant de la réponse en temps réel entre le joueur et la machine. Pour qu'il soutienne la construction d'apprentissages, le jeu doit offrir une histoire au joueur. Celle-ci doit être réaliste et faire l'objet d'un développement en séquences temporelles qui permettent au joueur d'être confronté à un degré de difficulté progressif. Il ne peut donc s'agir de jeu fondé sur le recours à des séquences «en boucles». Enfin, les jeux électroniques qui soutiennent l'apprentissage doivent permettre l'exercice d'un contrôle optimal du 
joueur, tant sur la durée de chacune des séquences de jeu que sur les paramètres qui définissent l'apparence de l'environnement virtuel auquel il est exposé (Griffiths, 2002; Wood, Griffiths, Chappell \& Davies, 2004). Le recours au jeu électronique doit donc permettre la sauvegarde des parties et leur réinitialisation au niveau et dans l'état de développement correspondant.

Ces éléments permettent de mettre en lumière l'appréciation différenciée et la lecture plus ou moins ludique de chacun des quatre logiciels ludo-éducatifs évalués par nos jeunes sujets. Au plan de la qualité technologique, du degré de sophistication des séquences, de la gradation des niveaux de difficulté, les deux logiciels produits pour l'environnement scolaire ne peuvent faire face avec succès aux deux logiciels de grande diffusion.

\section{Logiciels scolaires de type Ludique ou appropriation de logiciels ludiques pour fins scolaires?}

Les études portant sur l'utilisation des logiciels de jeu afin de soutenir l'apprentissage scolaire semblent suggérer une certaine efficacité de ces pratiques. Néanmoins ces travaux soulèvent aussi plusieurs écueils au plan pratique. Ainsi, la charge de travail exigée de la part des enseignants, notamment au plan de l'évaluation des propriétés didactiques des logiciels sélectionnés ainsi qu'en matière d'effets sur l'organisation des activités et du temps de classe semblent mener rapidement au désengagement des praticiens à cet égard. En clair, l'intégration scolaire du jeu électronique en tant que matériel didactique se trouve rapidement confrontée aux mêmes facteurs qui nuisent à l'intégration pédagogique des TIC dans les pays industrialisés. L'impact sur les conditions de gestion de l'enseignement ainsi que sur la gestion de classe en réduit fortement la probabilité d'utilisation (Jones, 2004; Larose, Grenon \& Palm, 2004; Mumtaz, 2000).

Une des conséquences de ce qui précède pour qu'il y ait intégration didactique du jeu électronique en classe, il faudrait que les logiciels aient été préalablement analysés au plan du rapport entre leurs contenus, ou les habiletés auxquelles ils font appel, et les compétences spécifiques aux programmes disciplinaires qu'ils doivent soutenir. La mise à la disposition des enseignants de guides pédagogiques identifiant, par exemple, les segments de différents jeux qui font appel à des savoirs ou à des compétences acquises ou utiles en contexte scolaire ainsi que la durée d'une séquence (quelques tableaux) pouvant être intégrée à l'intérieur d'une période normale d'enseignement, faciliterait cette intégration. Par ailleurs, les jeux vidéos sont souvent disponibles de façon concomitante sur plusieurs types de plates-formes. Le faible coût d'achat de ces plates-formes, souvent "déclassées», peut permettre de disposer d'équipements en classe, là où la perspective du déplacement vers le laboratoire d'informatique peut occasionner des problèmes d'ordre logistique aux enseignants. 


\section{Conclusion}

Il existe un discours «fort» en éducation, notamment depuis le démarrage des principales réformes curriculaires en Amérique du Nord et en Europe de l'Ouest. Ce discours met l'emphase sur l'importance de recourir à des contextes d'enseignement et d'apprentissage authentiques afin d'arrimer les pratiques enseignantes au vécu ainsi qu'à la réalité des élèves. On peut considérer qu’il en va de l'arrimage des pratiques de recours aux médias électroniques comme de celui des autres dimensions de la pratique enseignante à la réalité sociale des élèves. Si la base principale du hiatus entre les profils de recours à l'informatique à la maison et à l'école réside dans le type de tâche effectuée à l'aide de l'ordinateur et si une partie de cette distance réside dans la part que prend le recours au jeu lorsque l'enfant utilise l'ordinateur à la maison, on peut penser que la définition même de ce qu'est une tâche de type ludique pour un élève doit être prise en considération. Lorsqu'il pratique un jeu, un joueur exerce un contrôle optimal non seulement sur la nature de la tâche elle-même, mais sur le moment où il s'y adonne ainsi que sur la durée de l'activité. La planification de l'enseignement en contexte scolaire ne permet pas l'exercice optimal d'un tel contrôle. Il y a donc là matière à réflexion sur le rôle et la place des activités ludiques par rapport à l'apprentissage scolaire.

S'il est possible de reproduire les conditions et les objets des pratiques ludiques des jeunes au sein de certains contextes d'apprentissage, on peut très certainement considérer que certaines de leurs pratiques de recours au jeu électronique offrent un potentiel de transfert ou de soutien à l'apprentissage scolaire. Pour y arriver, encore faut-il connaître le profil réel de ces pratiques d'une part et, d'autre part, démontrer aux enseignants que ces dernières ne sont pas incompatibles avec l'organisation de l'enseignement. Pour réaliser ce second objectif, il est nécessaire de connaittre les représentations que les praticiens ont au regard du jeu électronique, peu importe son support, et de ses effets sur l'enfant d'âge scolaire. Peu de recherches en sciences de l'éducation se sont penchées sur ces deux questions. Pourtant, la documentation de ces deux univers s'avère un incontournable si on désire réduire le nouvel écart numérique qui semble éloigner une fois de plus le vécu social et le vécu scolaire de l'élève. C'est à cette tâche que notre programme de recherche désire s'atteler.

\section{Notes}

1 Larose, F., Lenoir, Y., Bédard, J., Hasni, A., Karsenti, T., Kalubi, J.-C., Morin, M.-P., Terrisse, B. \& Trudel, M. (2004-2007). Le rapport des enseignants du préscolaire et du primaire aux pratiques d'utilisation des médias électroniques de leurs élèves: Obstacle ou instrumentation pédagogique? Conseil de recherches en sciences humaines du Canada (CRSH) (Programme de recherche ordinaire).

2 http://c-rdi.qc.ca/produits/liste_titre.asp?submit=ok

3 Projet SEUR: http://www.seur.qc.ca/info.htm 
4 Cet indice tient compte des variables de pondération suivantes: revenu familial brut cumulatif déclaré, scolarité de la mère, stabilité d'emploi du père et monoparentalité. (Statistique Canada, 2004b).

5 Afin de ne pas allourdir indûment le texte, nous n'y intègrerons pas les plans factoriels produits lors de l'analyse statistique textuelle.

\section{Références bibliographiques}

Aleven, V., Stahl, E., Schworm, S., Fischer, F. \& Wallace, R. (2003). Help seeking and help design in interactive learning environments. Review of Educational Research, 73 (3), 277320.

Anand, S. \& Krosnick, J. A. (2005). Demographic predictors of media use among infants, toddlers, and preschoolers. American Behavioral Scientist, 48 (5), 539-561.

Andersen, E. B. (1990). The statistical analysis of categorical data. Heidelberg: Springer Verlag.

British Educational Communication and Technology Agency (2001). Becta's Computer Games in Education Project Report. Londres, UK: British Educational Communication and Technology Agency (BECTA).

Bickham, D. S., Vanderwater, E. A., Huston, A. C., Lee, J. H., Caplovitz, A. G. \& Wright, J. C. (2003). Predictors of children's electronic media use: An examination of three ethnic groups. Media Psychology, 5 (2), 107-137.

Colley, A. \& Comber, C. (2003). Age and gender differences in computer use and attitudes among secondary school students: what has changed? Educational Research, 45 (2), 155-165.

Durkin, K. \& Barber, B. (2002). Not so doomed: computer game play and positive adolescent development. Applied Developmental Psychology, 23 (3), 373-392.

Frick, P. J., Christian, R.E. \& Wooton, J. M. (1999). Age trends in the association between parenting practices and conduct problems. Behavior Modification, 23 (1), 106-128.

Gentile, D. A. \& Walsh, D. A. (2002). A normative study of family media habits. Journal of Applied Developmental Psychology, 23 (2), 157-178.

Giegler, H. \& Klein, H. (1994). Correspondence analysis of textual data from personal advertisements. In M. Greenacre \& J. Blasius (dir.), Correspondence Analysis in the Social Sciences (pp. 283-301). New York, N.J.: Academic Press.

Gouvernement du Québec (2004). Indice de milieu socio-économique par école. Indice du seuil de faible revenu par école. Données 2003-2004. Québec: Ministère de l'éducation, Direction de la recherche, des statistiques et des indicateurs. Document téléaccessible. Online: [http://www.meq.gouv.qc.ca/stat/Indice_defav/index_ind_def.htm].

Grenon, V., Larose, F., Couture, M. \& Desforges, M.-C. (2003). Les enfants à risque de difficultés d'adaptation sociale et scolaire et les médias électroniques: facteurs de risque ou facteurs de protection? In F. Larose (dir.), Difficulté d'adaptation sociale ou scolaire et intervention éducative (pp. 139-156). Sherbrooke: Éditions du CRP.

Griffiths, M. D. (2002). The educational benefits of videogames. Education and Health, 20 (1), 47-51.

Heiser, W. J. \& Meulman, J. J. (1994). Homogeneity analysis: exploring the distribution of variables and their nonlinear relationships. In M. Greenacre \& J. Blasius (dir.), Correspondence analysis in social sciences (pp. 179-209). San Diego: Academic Press.

Hong, J. C. \& Liu, M. C. (2003). A study on thinking strategy between experts and novices of computer games. Computers in Human Behavior, 19 (2), 245-258.

Hooper, S. (2003). The effects of persistence and small group interaction during computerbased instruction. Computers in Human Behavior, 19 (2), 211-220.

Ivory, J. D. (2001). Video games and the elusive search for their effects on children: An assessment of twenty years of research. Washington, DC: Communication présentée au congrès annuel de l'Education in Journalism and Mass Communication, Août 2001. 
Jones, A. (2004). A review of the research literature on barriers to the uptake of ICT by teachers. Londres, UK: British Educational Communication and Technology Agency (BECTA).

Klopfer, E., Yoon, S \& Rivas, L. (2004). Comparative analysis of Palm and waerable computers for participatory simulations. Journal of Computer Assisted Learning, 20 (5), 347-359.

Kroesbergen, E. H. \& Van Luit, J. E. H. (2003). Mathematics interventions for children with special educational needs - A meta-analysis. Remedial and Special Education, 24 (2), $97-$ 114.

Larose, F., Grenon, V. \& Palm, S. (2004). Enquête sur l'état des pratiques d'appropriation et de mise en cuvre des ressources informatiques par les enseignantes et les enseignants du Québec. Volume 1: L'enquête par questionnaire. Sherbrooke, Québec: Université de Sherbrooke: Centre de recherche sur l'intervention éducative / Ministère de l'éducation, direction des ressources didactiques. Document téléaccessible.

Online: [http://www.educ.usherb.ca/crie/enligne/diffusion.htm].

Larose, F., Grenon, V., Pearson, M., Morin, J.-F. \& Lenoir, Y. (2004). Les facteurs sociologiques et pédagogiques qui affectent les pratiques des enseignants du primaire au regard de l'informatique scolaire. In J. F. Desbiens, J. F. Cardin \& D. Martin (dir.), Intégrer les TIC dans l'activité enseignante: Quelle formation? Quels savoirs? Quelle pédagogie? (pp. 59-80). Québec: Presses de l'Université Laval.

Larose, F., Lenoir, Y. \& Karsenti, T. (2002). À quoi peuvent bien servir les TIC en enseignement? In F. Larose \& T. Karsenti (Dir.), La place des TIC en formation initiale et continue à l'enseignement (pp. 27-52). Sherbrooke, Québec: Éditions du CRP.

Larose, F., Lenoir, Y., Karsenti, T. \& Grenon, V. (2002). Les facteurs sous-jacents au transfert des compétences informatiques construites par les futurs maîtres du primaire sur le plan de l'intervention éducative. Revue des sciences de l'éducation, XXVIII (2), 263-285.

Lebart, L., Salem, A. \& Berry, L. (1997). Exploring Textual Data. Dordrecht: Kluwer.

Linebarger, D. L. (2000). Summative evaluation of Between the Lions: A final report to WGBH Educational Foundation. Kansas City, KS: University of Kansas, Juniper Gardens Children's Project. Document téléaccessible. Online: [http://pbskids.org/lions/about/report/BTL_Report.pdf].

Lou, Y. P., Abrami, P. C. \& d'Apollonia, S. (2001). Small group and individual learning with technology: A meta-analysis. Review of Educational Research, 71 (3), 449-521.

Lucas, K. \& Sherry, J.L. (2004). Sex differences in video game play: A communication-based explanation. Communication Research, 31 (5), 499-523.

Margolis, J.L., Nussbaum, M., Rodriguez, P. \& Rosas, R. (2004). Methodology for evaluating a novel education technology: a case study of handheld video games in Chile. Computers and Education, 44 (3), (sous presse). Document téléaccessible.

Online: [http://www.vtie.nuim.ie/vtie/staff/041011-LR-MethodOfEval.pdf].

Mathes, P. G., Torgesen, J. K. \& Allor, J. H. (2001). The effects of peer-assisted literacy strategies for First-Grade Readers with and without additional computer-assisted instruction in phonological awareness. American Educational Research Journal, 38 (2), 371-410.

McFarlane, A., Sparrowhawk, A. \& Heald, Y. (2002). Report on the educational use of games. Cambridge, UK: Cambridge University, TEEM department for education and skills.

McLelland, D. \& Crawford, J. (2004). The Drumchapel Project: a study of ICT usage by school pupils and teachers in a secondary school in a deprived area of Glasgow. Journal of Librarianship and Information Science, 36 (2), 55-67.

Meulman, J. J., Van der Kooij, A. J. \& Heiser, W. J. (2004). Principal components analysis with nonlinear optimal scaling transformations for ordinal and nominal data. In D. Kaplan (Dir.), The Sage Handbook of Quantitative Methodology for the Social Sciences (pp. 4972). Thousand Oaks, CA: Sage Publications Inc.

Mistler-Jackson, M. \& Songer, N .B. (2000). Student motivation and Internet technology: Are students empowered to learn science? Journal of Research in Science Teaching, 37 (5), 459-479. 
Mumtaz, S. (2000). Factors affecting teachers' use of information and communications technology: a review of the literature. Journal of Information Technology for Teacher Education, 9 (3), 319-341.

Mumtaz, S. (2001). Children's enjoyment and perception of computer use in the home and the school. Computers and Education, 36 (4), 347-362.

Office for Standards in Education (2004). ICT in schools. The impact of government initiatives five years on. London, UK: Ofsted Publications Centre.

Rosas, R., Nussbaum, M., Cumsille, P., Marianov, V., Correa, M., Flores, P., Grau, V., Lagos, F., Lopez, X., Lopez, V., Rodriguez, P. \& Salinas, M. (2003). Beyond Nintendo: design and assessment of educational video games for first and second grade students. Computers and Education, 40 (1), 71-94.

Schott, G. \& Selwin, N. (2000). Examining the «male, antisocial» stereotype of high computer user. Journal of Educational Computing Research, 23 (3), 291-303.

Schwartz, R. S., Lederman, N. G. \& Crawford, B.A. (2004). Developing views of nature of science in an authentic context: An explicit approach to bridging the gap between nature of science and scientific inquiry. Science Education, 88 (4), 610-645.

Shyu, H. Y. (1999). Effects of media attributes in anchored instruction. Journal of Educational Computing Research, 21 (2), 119-139.

Sciadas, G. (2002). La fracture numérique au Canada. Ottawa: Statistique Canada.

Statistique Canada (2004a). Achats en ligne: magasinage des ménages sur Internet. Le Quotidien. Périodique électronique téléaccessible.

Online: [http://www.statcan.ca/Daily/Francais/040923/q040923a.htm].

Statistique Canada (2004b). Les seuils de faible revenu de 1994 à 2003 et les mesures de faible revenu de 1992 à 2001. Ottawa: Statistique Canada, Division de la statistique du revenu. Série de documents de recherche - Revenu.

Strasburger, V. C. \& Donnerstein, E. (1999). Children, adolescents, and the media: Issues and solutions. Pediatrics, 103, 129-139.

Subrahmanyam, K., Kraut, R. E., Greenfield, P .M. \& Gross, E. F. (2000). The impact of home computer use on children's activities and development. Future of Children, 10 (2), 123-144.

Ungerleider, C. \& Burns, T. (2003). Étude systématique de l'efficacité et de l'efficience des TIC en réseau dans le domaine de l'éducation. Toronto: Conseil des ministres de l'éducation du Canada (CMEC). Rapport de recherche.

Weinberger, A., Fischer, F. \& Mandl, H. (2003). Collaborative knowledge construction in computer-mediated communication: Effects of cooperation scripts on acquisition of application-oriented knowledge. Zeitschrift fur Psychologie - Journal of Psychology, 211 (2), 86-97.

Wikipedia (2004a). Wikipedia, the free encyclopedia. Document téléaccessible. Online: [http://fr.wikipedia.org/wiki/Console_de_jeux_vid\%C3\%A9o].

Wikipedia (2004b). Wikipedia, the free encyclopedia. Document téléaccessible. Online: [http://en.wikipedia.org/wiki/Video_game_genres].

Wood, R. T. A., Griffiths, M. D., Chappell, D. \& Davies, M. N. O. (2004). The structural characteristics of video games: A psycho-structural analysis. CyberPsychology, 7 (1), 1-10.

Young, J. \& Upitis, R. (1999). The microworld of Phoenix Quest: social and cognitive considerations. Education and Information Technologies 4 (4), 391-408.

Mots clés: Intégration des TIC, jeu électronique, apprentissage en contexte authentique, pratiques sociales de référence, enseignement au secondaire 


\section{Die Beziehung von Sekundarschülern zu elektronischen Spielen und die Anwendung von Lernspiel-Software im Unterricht}

\section{Zusammenfassung}

In diesem Beitrag stellen wir die Ergebnisse einer Sondierungsstudie, die in einer Sekundarschule durchgeführt wurde, vor. Dabei wurden die Benutzer/innengewohnheiten von Spielsoftware getestet. Auf der Basis der vorhandenen wissenschaftlichen Dokumentation in Bezug auf den Zugang zu elektronischen Spielen dokumentieren wir sowohl das Benutzerprofil der Jugendlichen im privaten Bereich als auch ihre Wahrnehmung bezüglich dem Einsatz von Informatik inkl. elektronischen Lernspielen im Unterricht. Darauf folgt eine Bewertung von vier Software-Produkten, die vom Erziehungsminister empfohlen wurden, durch die Schülerinnen und Schüler. Wir haben auch recherchiert, was in den Augen der Jugendlichen eine sowohl interessante und als auch lehrreiche Software auszeichnet. Zum Schluss bringen wir Vorschläge für eine Integration vom Spielsoftware-Umfeld in die Schulstunden, dies mit dem Ziel, die Kluft zwischen Schulgeschehen und dem Verhalten der Schülerinnen und Schüler in ihrer Freizeit zu verringern.

Schlagworte: Integration von Lernspielen ; Spielsoftware ; Lernen im authentischen Umfeld ; Freizeitverhalten ; Unterricht auf der Sekundarstufe

\section{Gli allievi delle scuole secondarie, i giochi elettronici e I'utilizzazione didattica di programmi ludico-educativi}

\section{Riassunto}

In questo articolo presentiamo i risultati di una ricerca condotta fra gli allievi delle scuole secondarie per mettere a fuoco le pratiche d'uso dell'informatica in contesti ludici. Dopo aver passato in rassegna la letteratura scientifica sull'esposizione dei giovani ai giochi elettronici, descriviamo sia il loro profilo relativamente all'uso domestico di tali giochi sia le loro rappresentazioni dell'applicazione dell'informatica nel contesto scolastico, compresa la dimensione ludico-educativa. Di seguito analizziamo quattro programmi raccomandati dal Ministero dell'educazione e dello sport così da identificare, dal punto di vista dei giovani, quali sono le caratteristiche di un programma che sia ad un tempo interessante ed tale da favorire l'apprendimento scolastico. Concludiamo con alcune proposte di integrazione di programmi ludici in classe al fine di ridurre lo scarto tra vissuto scolastico e pratiche sociali degli allievi.

Parole chiave: Integrazione delle TIC, giochi elettronici, apprendimento in contesti autentici, pratiche sociali, insegnamento secondario 


\section{Secondary school students' relationship to electronic games, and the use of educational gameware in tuition}

\section{Abstract}

This article reports on results of a preliminary study which has been carried out with a sample of secondary school students concerning their habits in using a computer game. After reviewing the scientific literature on the exposition of students to electronic games in and out of school, we describe the profile of young people in how they use gameware, including educational programs, in both private and school contexts. We then report about the results of a study in which students assessed four educational e-games which had been supported by the Ministry for Education. Further results of students' estimations about what makes game software educational and interesting are reported. In order to reduce the gap of students between experiences within and outside of school, we conclude our contribution with suggestions on how gameware, and ICT in general, can be integrated in tuition and school.

Key words: Integration of ICT; electronic game; learning in an authentic context; social reference practices; high school teaching. 